\title{
Energy and Thermodynamic Considerations Involving Electromagnetic Zero-Point Radiation
}

\author{
Daniel C. Cole \\ Boston University, Dept. of Manufacturing Engineering, 15 St. Mary's Street, Boston, MA 02215 \\ Phone: (617) 353-0432, Email: dccole@bu.edu
}

\begin{abstract}
There has been recent speculation and controversy regarding whether electromagnetic zero-point radiation might be the next candidate in the progression of plentiful energy sources, ranging, for example, from hydrodynamic, chemical, and nuclear energy sources. Certainly, however, extracting energy from the vacuum seems counter intuitive to most people. Here, these ideas are clarified, drawing on simple and common examples. Known properties of electromagnetic zeropoint energy are qualitatively discussed. An outlook on the success of utilizing this energy source is then discussed.
\end{abstract}

\section{INTRODUCTION}

The present article reviews some of our present understanding of the electromagnetic zero-point (ZP) fields, in particular regarding concepts in thermodynamics related to energy and heat extraction. Topics that will be touched on are (1) the relationship between the ZP fields and the nonzero temperature thermodynamic equilibrium situations, (2) the more general nonequilibrium case, (3) energy and heat extraction, (4) reversible and irreversible thermodynamic operations, (5) the connection of all of these ideas to conventional ideas on thermodynamics, (6) "restraints" on extracting heat and energy from electromagnetic ZP radiation, (7) a brief summary of our present understanding of many of the key properties of electromagnetic ZP fields, and (8) an outlook on making use of the $\mathrm{ZP}$ fields for energy extraction. The aim here will be to be fairly qualitative, as a number of articles exist that explain more of the details of these topics.

All of these topics will be treated from the viewpoint that the electromagnetic ZP fields are real. Certainly there are other viewpoints, including Schwinger's source theoretical viewpoint, but these viewpoints are all connected and are presently generally thought to be consistent (Milonni, 1994). Moreover, there are “... many observable consequences of the vacuum field, including spontaneous emission, the Lamb shift, the anomalous magnetic moment, van der Waals forces, and the fundamental laser linewidth, all of which may be attributed at least in part to the vacuum field." The viewpoint that the ZP fields are real certainly makes the thermodynamic discussion much easier and more natural and so shall be followed here.

Why the interest here? Well, if one does a rough estimate of the energy density associated with electromagnetic ZP energy, it is easy to show that, even with a very conservative estimate, the energy density must be incredibly large, namely, equal to or greater than nuclear energy densities (Feynman, 1973), (Misner, 1973). Can useful work be extracted from this apparent huge source? We will quickly see that the answer here is certainly, "Yes." Moreover, heat can be extracted, even if the initial temperature could be, in principle, at the temperature of absolute zero, $T=0$. At first thought this statement must appear quite contradictory, since how can one obtain heat from a system initially held at $T=0$ ? The example discussed in Sec. III should clarify the answer considerably.

Other questions that then naturally come to mind are, "If heat and useful work can be 'extracted' from the electromagnetic fields, exactly how much can be obtained?" After all, perhaps, for some reason, the allowed extracted magnitudes of energy are extremely small, despite the large energy density that exists. Perhaps some thermodynamic principle is violated if this energy was able to be accessed. As we will see, there are indeed some "restraints," for lack of a better word, on extracting energy from ZP energy. Nevertheless, these restraints are not an absolute barrier, by any means, to extracting heat and useful work, but rather they are simply restricting conditions. Moreover, I am unaware of any hard analysis that provides limits on the fraction or the total amount of energy that may be extracted from ZP energy in order to perform work, nor does the present article address this point.

\footnotetext{
${ }^{1}$ See (Milonni, 1994), p. 217.
} 
Answers to many of the other questions raised here will be discussed in the following sections using fairly qualitative terms. In addition, a number of examples will be discussed that hopefully make these answers easier to understand and accept, since the examples are taken directly from common experiences.

The outline of this article is as follows. The following section will describe some general information on electromagnetic ZP fields. The next section then spends some time on a simple example that illustrates energy and heat extraction at any temperature, including $T=0$, and then relates this example to other common energy sources. We will then turn to discuss some special properties of $\mathrm{ZP}$ radiation, and end in the final section of this article with what may be a surprising conclusion, but hopefully one that also helps to make more sense out of all of this material.

\section{GENERAL DISCUSSION ABOUT ELECTROMAGNETIC ZERO-POINT ENERGY}

Physicists' understanding about ZP energy in general has certainly had a very interesting history. For more details on this point, Chap. 1 in (Milonni, 1994) gives a very nice overview; (Kuhn, 1978) contains considerable historical and scientific information, while a source such as Chap. 4 in (Resnick, 1972) provides a more introductory discussion. Certainly, though, it can safely be said that our present common usage of the term "zero-point energy" is quite different than earlier notions of this term. The early ideas revolved very centrally around thermodynamics. Historically, the terminology of "zero-point" refers to the energy arising from fluctuating motion existing at the absolute zero of temperature, $T=0$, as opposed to the notion more commonly thought of today, namely, the "zeroth" or lowest quantum energy level of a system. Of course, these two concepts are related, but thinking in terms of the thermodynamic concept of the definition of ZP energy, provides an interesting and helpful insight into this concept of energy, as should become apparent in the course of this article.

For example, many physicists seem to have an intuitive perspective that there exists a quantum oriented "vacuum," consisting of the ZP fields associated with electromagnetism, as well as the quantum fields used for describing the annihilation and creation of all "particles," and that all of matter, including ourselves, are situated within this vacuum sea of fluctuating entities. This perspective is not the most economical means of picturing our situation, and perhaps most physicists would back off on this perspective if questioned deeply about it; nevertheless, it appears to be a prevailing view.

Hopefully the following brief discussion will help to explain this point more clearly. If one views the ZP fields as real quantities, which is a very legitimate perspective, then one can make the argument that there is little difference, aside from their thermodynamic properties, between "ZP fields" and these same fields, but at a different temperature. More specifically, consider a vessel containing electromagnetic radiation and an atomic system of particles (i.e., atoms, molecules, ions, and electrons), all at some equilibrium temperature $T>0$. Such a system does not possess "zero-point" energy, but rather possesses the thermodynamic energy associated with its $T>0$ equilibrium state. Moreover, if the system in the vessel is not in equilibrium, then it possesses neither ZP energy nor some other thermal energy, but rather an energetic state of nonequilibrium that will, in general, be changing with

\footnotetext{
${ }^{2}$ These references spend some time detailing the break that physicists traditionally have noted between "classical" physics and the observed phenomena in nature that led to the development of quantum mechanics. It should be emphasized, however, that physicists in the late $1800 \mathrm{~s}$ and early 1900s usually made the implicit assumption that classical physics does not allow fluctuating motion of classical charged particles and classical electromagnetic fields at a temperature of $T=0$. [Here, by "classical physics," I mean the behavior of classical charged particles interacting with classical electromagnetic radiation, via Maxwell's equations and the traditional electromagnetic Lorentz force, and where one assumes the relativistic version of Newton's second law of motion describes the equation of motion for classical charged particles (Rohrlich, 1965), \{Boyer, 1975).] Reference (Cole, 92a) contains more information on the changes that are necessary in the traditional arguments involving the compression of blackbody radiation (derivation of Wein's displacement law) when this physical assumption of no fluctuations is not imposed at $T=0$. References (Cole, 1990a) and (Cole, 1990b) discuss other related points that are often cited as a failure of classical physics, such as the ultraviolet catastrophe, infinite specific heat, and the third law of thermodynamics. References (Cole, 1990a), (Cole, 1992a) and (Cole, 1992b) analyze specific classical electrodynamic systems and provide derivations for the classical electromagnetic ZP spectrum, based on the thermodynamic definition of temperature. In this work care is taken to make sure that the implicit assumption that motion and fluctuations reduce to zero at $T=0$ is not made in the analysis, as occurred, sometimes inconsistently, at various points in the work by physicists in the early 1900s. Reference (Cole, 1993a) reviews some of this work and provides some qualitative incentives (e.g., Earnshaw's theorem) for these considerations. The work by Boyer in Refs. (Boyer, 1969a and \{Boyer, 1969b began much of the early and insightful thermodynamic analysis of these ideas. Section 8.12 in Ref. (Milonni, 1994) briefly discusses the resulting classical physical theory, usually called stochastic electrodynamics, and mentions some references, but does not really touch on the thermodynamic issues raised by allowing ZP motion for classical particles and fields.
} 
time, unless some sort of steady-state nonequilibrium condition is being maintained, such as by an imposed temperature gradient. Since nearly all sections of our universe are in nonequilibrium states, that at best only approximate equilibrium conditions, then the most economical viewpoint may be to not assume that ZP fields pervade our universe, but only in some sense they approximately do so.

Let us consider an idealistic case of only electromagnetic radiation present at some temperature $T$, with no other fields present. First, and not surprisingly, the radiation's statistical properties must be homogeneous and isotropic in space, and stationary in time for this idealized situation. An additional interesting symmetry holds for the $T=0$ case: Marshall (Marshall, 1963) and Boyer (Boyer, 1969a) showed that the electromagnetic ZP spectrum has a Lorentz invariant property associated with it, so that observers moving at constant velocities with respect to each other, each in a different inertial rest frame, both see the same ZP energy spectrum. In contrast, a radiation spectrum associated with a temperature $T>0$, is most definitely not invariant under Lorentz transformations.

In addition, a host of other very interesting properties have been identified for electromagnetic ZP radiation, as well as electromagnetic ZP plus Planckian radiation. For example, ZP radiation does not yield velocity-dependent forces and it gives rise to thermal effects associated with acceleration. These, and many other properties are discussed in Ref. (de la Peña, 1996) and in the references cited therein.

\section{EXAMPLES OF ENERGY AND HEAT EXTRACTION}

Here we will discuss a few examples intended to provide qualitative and intuitive answers to many of the questions raised at the beginning of this article. The examples will start with an incredibly simple example that, despite its simplicity, will hopefully shed light on these questions as well as more complicated problems involving ZP energy in quantum mechanical systems. More complicated examples will then be briefly mentioned. The first example is aimed at addressing and clarifying the notion of extracting useful work and/or heat at $T=0$, although, actually, the concepts apply at any temperature $T$.

Figure 1 shows a ball on a table with "bumpers" that are positioned to prevent the ball from rolling off. The radius of the ball is $R$, the height of the top surface of the table from the grgund is $H$, and the height of the bumpers is $h$. The system is designed to be in mechanical equilibrium, as there are ".... no unbalanced forces acting on any part of the system or on the system as a whole." Assuming that the ball and table are also in thermal equilibrium, meaning that ${ }^{3}$ " $\ldots$ there are no temperature differences between parts of the system or between the system and its surroundings," and that everything is also in chemical equilibrium, meaning that ${ }^{3}$ "... there are no chemical

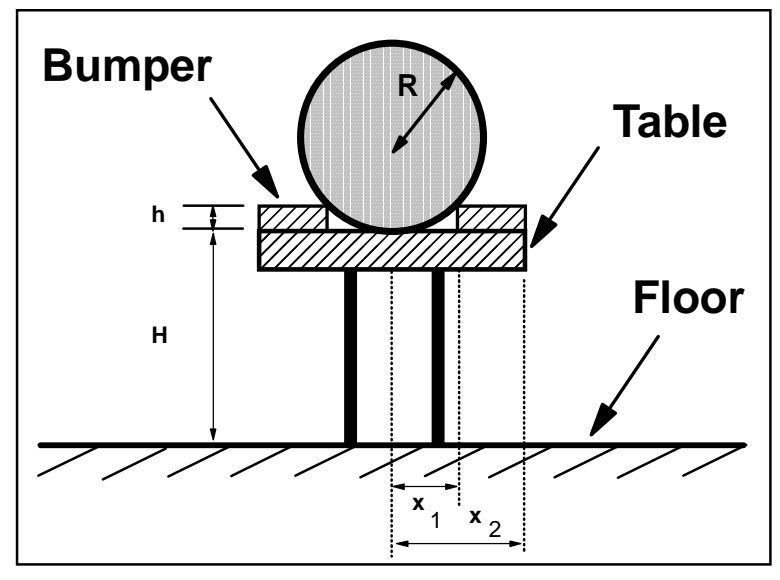

FIGURE 1. Illustration of a ball on a table, held in place by two supports, or bumpers, to keep the ball from rolling.

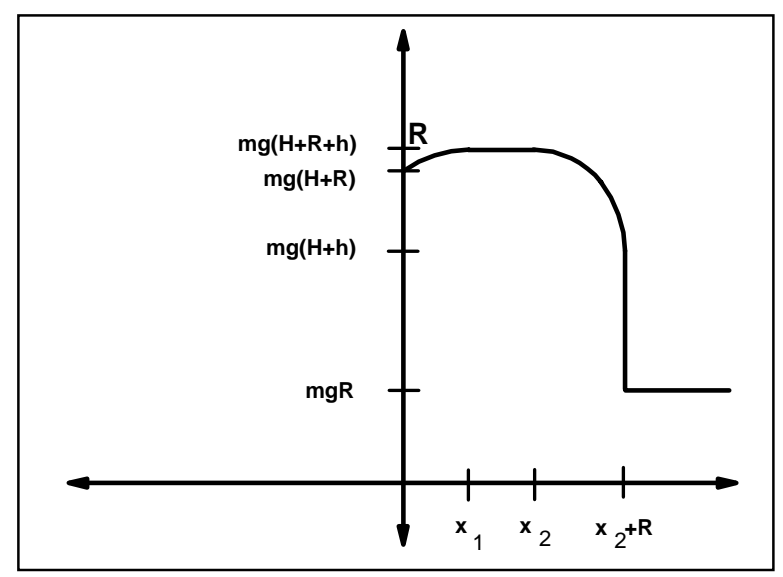

FIGURE 2. Plot of gravitational potential energy for the center of mass of the ball, as a function of position $\mathrm{x}$.

\footnotetext{
${ }^{3}$ See (Zemansky, 1981), p. 49.
} 
reactions within the system and no motion of any chemical constituent from one part of a system to another part," then the ball and table satisfy the condition of being in thermodynamic equilibrium. The system can be in thermodynamic equilibrium at any temperature, although the system as we know it may drastically change to a much different equilibrium state if too high a temperature is reached. In particular, then the equilibrium state may involve a melted ball and/or table, at which point the conditions of mechanical and chemical equilibrium must be reexamined.

Let us consider the ball to be a macroscopic object composed of microscopic entities, (e.g., atoms and molecules) with many degrees of freedom. Thus, we can think of the ball as containing internal energy of its own, depending on the equilibrium state of its internal degrees of freedom. Besides the table that the ball is in contact with, let us assume that there is electromagnetic radiation bathing the ball, table, and floor, and that initially all are in thermodynamic equilibrium with each other.

Figure 2 shows the most obvious component of the potential energy of this system, namely, a plot of mgy versus $x$, where $m$ is the mass of the ball, $g$ is the gravitational acceleration (assuming a uniform gravitational field), and $y$ is the height of the center of the ball from the floor. There certainly exist other potential energy sources, such as might result from a chemical decomposition of these structures, but we will not consider these aspects in the present example.

If the ball is slowly pushed to one side and rolled up and over, say, the right bumper, and then slowly lowered to the floor, such as by a means of mechanical pulleys, cords, and springs, then useful work could be done by the ball on another system. More specifically, the weight of the ball might be used to raise another mass via a pulley system, or used to compress a spring. However, because of the presence of the bumper, then in order for this work to be done, which equals $m g H$, a small amount of work, equal to $m g h$, must first be done on the system in Fig. 1. This energy barrier is closely related to the activation energy in chemical processes. If the movement of the ball is carried out very slowly, so that bumps and jarring of the structures do not occur, thereby activating internal vibrations within the ball and in the constraining structures, then the process is a thermodynamically reversible process, as the system can be restored back to its original state without any changes in the rest of the universe.

Of course, the above process is an idealized, hypothetical one, as are all reversible processes that are considered in thermodynamics. In practice, jarring and bumping of the ball, or friction in the ropes of the pulley, would result in the process being a thermodynamically irreversible process. Indeed, let us consider the extreme thermodynamically irreversible process where the ball is rolled over the bumper and simply dropped. Work equal to $m g h$ must be done on the ball in order to raise it over the bumper. As the ball falls, there are no constraining forces or structures for the ball to do work on. The ball will pick up speed as it falls. Upon landing on the floor, it will bounce; dissipative energy mechanisms will be activated involving internal vibrations in the ball and in the floor. The degree of the ball's elastic material composition will dictate how long the ball continues to bounce until dissipative forces at play have essentially dissipated the energy $m g(H+h)$.

Let us assume that the ball, table, and floor are all enclosed within a chamber with adiabatic walls, so that no heat flows out of the chamber during this entire process. Due to the adiabatic walls, and since the ball does no work on any external system during its free fall, the net gain in internal energy of the system must be equal to $+m g h$, due to the extra work put into the system initially when raising the ball to the top of the bumper. Consequently, when the floor, ball, and walls housing the system, have come to equilibrium with the electromagnetic radiation bathing the system, the temperature of the total system will have risen.

If one wished to calculate the entropy change of this system, it could be done by considering a reversible thermodynamic process that connects the initial and final equilibrium state arising from the irreversible thermodynamic process just discussed. One would need to calculate the change in caloric entropy given by

$$
S_{\mathrm{cal}, f}-S_{\mathrm{cal}, i}=\int_{i}^{f} \frac{d Q}{T},
$$


where $d Q$ is the heat flow into the system, and the integration is over any reversible path connecting the two states. The following sequence of reversible processes are convenient to consider. First, the ball could be slowly rolled up and over the right bumper and then slowly lowered to the floor. The constraining forces would then have done a net amount of work equal to $-m g H$ on the ball, or, rather, the ball would have done work on the pulley system of weights, or springs, that acted to slowly lower the ball, thereby changing the internal energy of the ball, table, and floor by a net amount of $-m g H$. In order to reversibly reach the final state of the system due to the irreversible process just discussed of dropping the ball, where the change in internal energy was $+m g h$, then one would need to reversibly add energy equal to $+m g(H+h)$ to the system. This could be accomplished by sequentially contacting the system with a series of heat reservoirs with infinitesimally increasing temperatures. The temperature of the system will then rise accordingly.

The previous example was incredibly simple, and of course very hypothetical, yet it contains many of the key features important for understanding how heat and energy can be extracted from ZP energy. While sitting on the table, the ball, table, and thermal radiation in the chamber could all have been, in principle, in equilibrium at the lowest temperature possible, namely, at absolute zero. Yet, by adding a slight amount of energy equal to $m g h$ to raise the ball to the height of the bumpers, one can then have the ball do external work $m g(h+H)$ by slowly lowering the ball. As in Ref. (Cole, 1993b), let us refer to this process of having the system do positive work by the term of "energy extraction." If instead we had the ball fall freely, so that no external work could be done while the ball was falling, then the resulting increase in kinetic energy of the ball, equal to $m g(h+H)$, must be converted into thermal energy associated with (1) the center of mass of the ball, (2) the internal degrees of freedom of the ball, and (3) the radiation in the chamber, thereby increasing the temperature of the system. If we then relax the adiabatic nature of the chamber walls, such as by poking a pinhole in the wall, then a net radiation energy could flow out of the chamber, assuming that the temperature outside the chamber is smaller than the temperature inside the chamber. As in Ref. (Cole, 1993b), let us refer to this process as being "heat extraction," which should be distinguished from the previous case of "energy extraction."

The examples discussed in Ref. (Cole, 1993b) dealt with more complicated systems that scientists typically associate with electromagnetic ZP energy, namely, plates attracted by the Casimir force, as well as atoms and molecules attracted by the van der Waals force. The same sort of operations were discussed, however, as in the example above. The point that, hopefully, the present example makes more strongly, is that energy and heat extraction can apply to even the simplest of systems, such as a ball falling off of a table, and does not require the consideration of complicated Casimir and van der Waals forces. There is nothing mysterious going on here; energy arising from a system initially in thermodynamic equilibrium at $T=0$, or $T>0$, simply requires the change of configuration forces acting on the system. Energy is still certainly conserved, but the system and universe have irreversibly changed, just as would occur in, for example, the case of a waterfall being formed due to a damn being opened.

Perhaps the above discussion alleviates concern about energy conservation. However, what about the second law? According to the Kelvin-Planck statement of the second law of thermodynamics, "No process is possible whose sole result is the absorption of heat from a reservoir and the conversion of this heat into work." At first glance, the previous process where heat was generated might appear as though it violates this law. However, such is not the case, since the generation of heat was not the only sole result of the process. Rather, the relationship between the ball and the table was irreversibly changed. If we use the height $y$ as an indicator of the internal energy associated with the ball due to the gravitational potential energy, the initial state of the ball and table had $y=H+R$, but at the end the height was $y=R$. The Kelvin-Planck statement only refers to physical processes where the state of the system does not change, which was not the case here. Reference (Cole, 1995) discusses a similar point regarding the possible influence of electromagnetic ZP radiation on astrophysical phenomena observed in nature.

There are a host of other subtle points, questions, and possible objections that one might raise to the previous example, particularly because the absolute zero of temperature is a hypothetical, idealized state that physicists believe can never be fully reached in practice. Nevertheless, the main points of this example should be fairly evident: if a system is taken out of equilibrium, in particular, mechanical, thermal, or chemical equilibrium, then

\footnotetext{
${ }^{4}$ See Ref. (Zemansky, 1981), p. 147.
} 
thermodynamic equilibrium conditions cannot be realized. Instead, new degrees of freedom then become available to the system, thereby enabling work to be done by the system and for heat to flow. Often only a relatively very small amount of energy is required (e.g., $m g h)$ in comparison to the energy realized (e.g., $m g H)$ in order for this to happen, as occurs in the case of a hole being made in a damn holding back a huge lake of water, or the puncture of a container filled with high pressurized gas, or perhaps a tiny spark needed to ignite a keg of gunpowder. Chemical reactions, such as occurs in a chemical battery, the lighting of a match, or, indeed, the explosion resulting from igniting a highly combustible material, are enormously more complicated than our simple example, yet the same basic ideas still remain.

\section{OTHER THERMODYNAMIC POINTS TO CONSIDER}

Here let us review some key thermodynamic properties that have been analyzed in some detail in the past and that have some bearing on energy and heat extraction. First, the second law of thermodynamics enables the following definition to be established for the ratio of two Kelvin temperatures $T_{a}$ and $T_{b}$ :

$$
\frac{Q_{R}\left(\text { between } \sigma_{\mathrm{I}} \text { and } \sigma_{\mathrm{II}} \text { at } T_{a}\right)}{Q_{R}\left(\text { between } \sigma_{\mathrm{I}} \text { and } \sigma_{\mathrm{II}} \text { at } T_{b}\right)}=\frac{T_{a}}{T_{b}},
$$

where the numerator on the left side is the heat flow into a system during an isothermal reversible process at temperature $T_{a}$, between two adiabatic surfaces indicated by $\sigma_{\mathrm{I}}$ and $\sigma_{\mathrm{II}}$. The denominator is the similar quantity, but now the heat flow is different because the reversible, isothermal process is carried out at temperature $T_{b}$. We immediately see that if one carries out any such reversible process at $T=0$, then no heat can flow either into or out of the system. Thus, if two Casimir plates, or two atoms or molecules interacting via van der Waals forces, are slowly moved toward or away from each other at $T=0$, then no heat can flow into or out of this system. This behavior was analyzed in some detail for several different types of systems in Refs. (Cole, 1990a), (Cole, 1990b), (Cole, 1992a), and (Cole, 1992b), resulting in the functional form of classical electromagnetic ZP radiation to be deduced in order for this property to hold.

As mentioned in the introduction to this article, there are indeed some "restraints" for extracting energy and heat at, or near, the temperature of $T=0$. The above point is one such restraint for heat extraction in that as long as one considers only reversible, or approximately reversible thermodynamic processes, then either none, or very little, heat will flow into or out of a system, no matter how drastically one perturbs the system. This behavior should be a very general property for any system at or near $T=0$. If, however, a system is at some temperature $T>0$, then heat will always flow in general for a reversible isothermal process, although the heat flow will become quite small as $T$ approaches absolute zero. Regarding work being done, yes, a system at $T=0$ can do work during a reversible thermodynamic process.

In contrast, if an irreversible thermodynamic process is considered, such as the example discussed earlier here, or the examples in Refs. (Cole, 1993b) and (Cole, 1995), then both heat can flow and work can be done; indeed, the magnitude of the heat flow and work done can be quite enormous.

Another "restraint" also exists for energy and heat extraction at or near $T=0$. This restraint involves the third law of thermodynamics, as discussed in some detail in Ref. (Cole, 1990b) (see Sec. VIII), as well as in Ref. (Cole 1990a). Specifically, the unattainability statement of the third law of thermodynamics is traditionally expressed as, "By no finite series of processes is the absolute zero attainable." Experimentalists indeed find this behavior to hold in that ${ }^{5}$ " $\ldots$ the fundamental feature of all cooling processes is that the lower the temperature achieved, the more difficult it is to go lower."

Thus, we have identified two mechanisms by which physical systems exhibit some "resistance" to parting with their "store" of fluctuating energy at $T=0:$ (1) we can never quite attain the $T=0$ state through any finite series of

\footnotetext{
${ }^{5}$ See Ref. (Zemansky, 1981), p. 515.
} 
processes, and (2) even if we did achieve $T=0$, then no heat can flow during reversible isothermal processes at $T=0$.

\section{CONCLUDING REMARKS}

This article addressed some of the conceptual problems associated with extracting heat and energy from electromagnetic ZP radiation. Clearly there should be no doubt that, yes, heat can be extracted from electromagnetic $\mathrm{ZP}$ radiation, via taking a system out of thermodynamic equilibrium and allowing a thermodynamic irreversible operation to take place. The Casimir plate example described in Ref. (Cole, 1993b) clearly illustrates this point, as does the even simpler example of the ball discussed in the present article. Moreover, yes, work can be done by a system at $T=0$, either during a reversible or an irreversible thermodynamic process. Some of these processes may require the input to the system of an activation energy to get the process started, as in the simple example discussed here, but in principle the activation energy can be quite small compared with the resulting work that is done and the heat that flows in the subsequent process. Since the second law of thermodynamics cannot be violated, then a net change in the state of the system must occur during this energy and heat extraction process. The situation is precisely analogous to obtaining energy during a chemical combustion process or a nuclear reaction; the input of a small amount of energy, such as the striking of a match to burn a pile of wood, or the initiation of a controlled chain reaction in a nuclear reactor, can result in considerable more energy than was used to start the process. The end result, however, is that the entropy for the entire system and universe increases as such "fuel sources" become used up, resulting in these sources of energy no longer possessing the same potential to do work. Likewise with our previous example, once the ball falls to the floor and either does work on the way down and/or else eventually dissipates most of its kinetic energy, then the thermodynamic state of the system changes (height changes and temperature may change, depending on the process) and the energy of $m g H$ can no longer be extracted again.

Also mentioned here were two interesting barriers to extracting the energy stored in the ZP state of any system. However, by taking the system out of thermodynamic equilibrium, then heat and work done can certainly still be extracted from a system.

I will end this article by making the following point. Much of the common energy sources we are familiar with, such as chemical combustion, electric batteries, and perhaps even nuclear energy, may already be, at least in some sense, in part due to extracting energy from zero-point fields. For example, it is fairlywell accepted now that vacuum fields contribute to atomic stability, spontaneous emission, laser behavior, etc. Thus, in a sense, the electromagnetic ZP field may already be viewed as a contributing source to various chemical processes. Perhaps the ZP fields associated with the strong interaction in nuclear physics processes may also be viewed as a contributor to already well known energy extraction processes.

Clearly, the previous point is somewhat speculative and requires further investigation to be more definitive. However, if this point is true, then does that leave us with any hope for new methods on obtaining energy from the apparent vast store of energy within ZP fields, or are such methods simply already well known methods, such as the means for extracting energy from a chemical battery? I don't think we really knows the answer to this question yet. My own suspicion is that the answer is, yes, there will be new and novel ways of extracting energy, although how plentiful the energy will be in comparison to already known sources is entirely speculative. Certainly, however, the incentive exists here to explore the properties of the vacuum more, such as is being done in cavity QED, to understand better how the states at and near $T=0$ can be controlled, to better understand the thermodynamics of such processes, including possible restrictions, and to better understand the relationship of known energy extraction processes to the apparent energy available in ZP fields.

\section{REFERENCES}

Boyer, T. H., Phys. Rev. 182, 1374-1383 (1969a).

Boyer, T. H., Phys. Rev. 186, 1304-1318 (1969b).

\footnotetext{
${ }^{6}$ See, for example, p. 81 in Ref. (Milonni, 1994), as well as numerous other places throughout this text.
} 
Boyer, T. H., Phys. Rev. D 11, 790-808 (1975).

Cole, D. C., Phys. Rev. A 42, 1847-1862 (1990a).

Cole, D. C., Phys. Rev. A 42, 7006-7024 (1990b).

Cole, D. C., Phys. Rev. A 45, 8471-8489 (1992a).

Cole, D. C., Phys. Rev. A 45, 8953-8956 (1992b).

Cole, D. C., "Reviewing and Extending Some Recent Work on Stochastic Electrodynamics," in Essays on Formal Aspects of Electromagnetic Theory, edited by A. Lakhtakia, Singapore, World Scientific, 1993a, pp. 501532.

Cole, D. C. and Puthoff, H. E., Phys. Rev. E 48, 1562-1565 (1993b).

Cole, D. C., Phys. Rev. E 51, 1663-1674 (1995).

de la Peña, L. and Cetto, A. M., The Quantum Dice. An Introduction to Stochastic Electrodynamics, Boston, Kluwer, 1996.

Feynman, R. P. and Hibbs, A. R., Quantum Mechanics and Path Integrals, New York, McGraw-Hill, 1965, p. 245.

Kuhn, T. S., Black-Body Theory and the Quantum Discontinuity, 1894-1912, New York, Oxford University Press, 1978.

Marshall, T. W., Proc. Roy. Soc. A 276, 475-491 (1963).

Milonni, P. W., The Quantum Vacuum. An Introduction to Quantum Electrodynamics, San Diego, Academic Press, 1994.

Misner, C. W., Thorne, K. S., and Wheeler, J. A., Gravitation, San Francisco, Freeman, 1973, p. $1202 \mathrm{ff}$.

Resnick, R., Basic Concepts in Relativity and Early Quantum Theory, New York, John Wiley, 1972.

Rohrlich, F., Classical Charged Particles, Reading, Addison-Wesley, 1965.

Zemansky, M. W. and Dittman, R. H., Heat and Thermodynamics, 6th ed., New York, McGraw-Hill, 1981. 